\title{
Primary pancreatic cystadenocarcinoma with ovarian metastases in a 38-year-old female: case report
}

\author{
Shun-Da Wang, Meng-Hua Dai, Yu-Pei Zhao \\ Department of General Surgery, Peking Union Medical College Hospital, Chinese Academy of Medical Sciences and Peking Union Medical \\ College, Beijing, China \\ Correspondence to: Dr. Prof. Meng-Hua Dai, MD; Dr. Prof. Yu-Pei Zhao, MD. Department of General Surgery, Peking Union Medical College \\ Hospital, Chinese Academy of Medical Sciences and Peking Union Medical College, Beijing 100730, China. \\ Email: Daimh@pumch.cn; zhao8028@263.net.
}

\begin{abstract}
Pancreatic cystadenocarcinoma with ovarian metastases is rare and easily misdiagnosed as primary ovarian cancers. Here we report 38-year-old female manifested tumors in pancreas and ovary concurrently, which was difficult to distinguish the primary site. She was admitted to hospital because of abdominal distension and a palpable mass in the lower abdomen. Abdominal ultrasound showed a lesion in pancreas and two masses in bilateral ovaries. Computed tomography (CT) revealed the hypo-enhancing pancreatic mass and the large pelvic lesion simultaneously. The largest cross-sectional of the right mass was $12 \times 15.1 \mathrm{~cm}$ and $15.4 \times 18.3 \mathrm{~cm}$ for the left side, probably malignant lesions. In addition, the level of the serum CA19-9 and CA125 were higher than the normal level. Positron emission tomography CT (PETCT) revealed there might be the cystadenocarcinoma in the pancreatic tail with multiple metastatic lesions implanted in the pelvic. After comprehensive examination, she received bilateral salpingo-oophorectomy and biopsy of the pancreatic tumor. The pathological finding revealed that it was pancreatic cystadenocarcinoma with ovarian metastases. Postoperatively, she received the chemotherapy and the follow-up continued for 26 months until she died. This case reminded doctors that pancreatic primaries should be paid attention when dealing with metastatic ovarian malignancies although it was rare. Choosing effective diagnostic method and timely surgical intervention are essential to improve prognosis.
\end{abstract}

Keywords: Ovary; metastasis; ovarian metastases; pancreatic cystadenocarcinoma; case report

Submitted Jun 19, 2020. Accepted for publication Oct 28, 2020.

doi: $10.21037 /$ tcr-20-2361

View this article at: http://dx.doi.org/10.21037/tcr-20-2361

\section{Introduction}

Pancreatic cancer is the fourth common cause of cancerrelated death and has pessimistic long-term survival (1). Among all of the pathological type of pancreatic cancer, pancreatic cystadenocarcinomas usually tend to metastasis to areas such as the liver, lung, spleen, bone and distant lymph nodes (2). However, ovarian metastases in female patients are very rare in clinical practice, difficult to early diagnosis, and usually miss the surgical opportunity. They are easily misdiagnosed as primary ovarian carcinomas. And the possibility of metastatic cancer needs to be considered when encountering lesions that appear simultaneously in the pancreas and ovary. Here we report a female case finally diagnosed with pancreatic cystadenocarcinoma with ovarian metastases. We present the following case in accordance with the CARE reporting checklist (3) (available at http:// dx.doi.org/10.21037/tcr-20-2361).

\section{Case presentation}

A 38-year-old woman became aware of abdominal distension and a palpable mass in the lower abdomen for 2 months. She denied the abdominal pain, diarrhea, and dyspepsia. And no significant weight loss was observed in the past 2 months. The patient denied any previous 

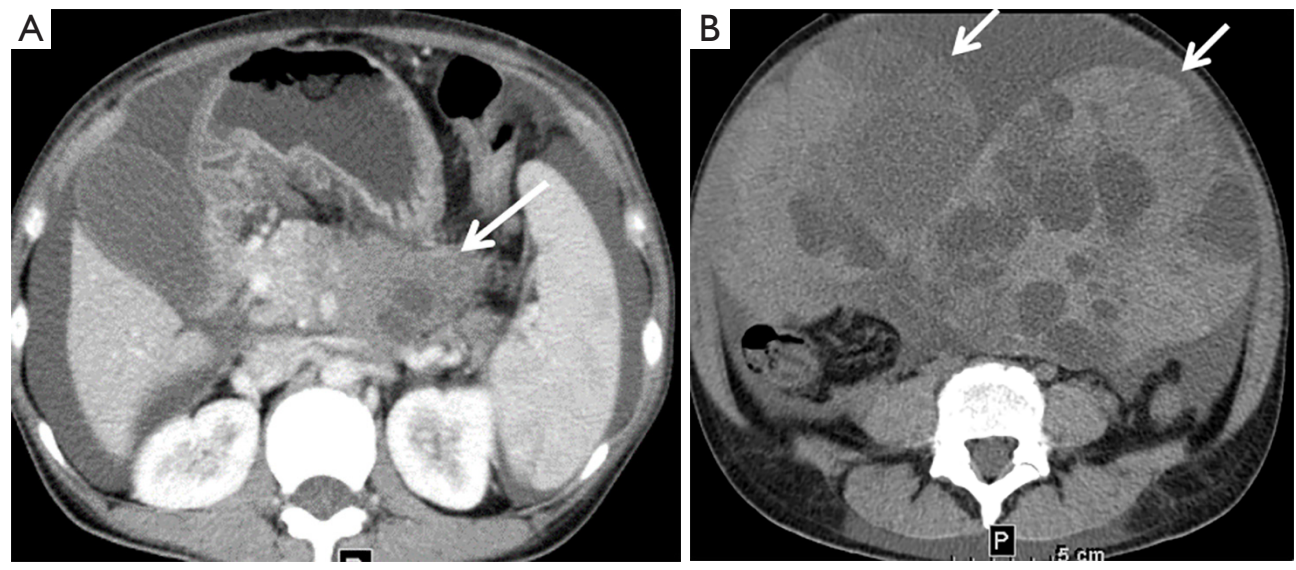

Figure 1 Abdominal CT revealed the lesions both in pancreas and ovary. (A) Abdominal CT demonstrated the hypo-enhancing pancreatic mass with necrosis and vascular invasion (white arrow); (B) enhanced CT imaging of the pelvic cavity showed that cystic-solid masses originated from the ovarian, protruding to the abdomen. The size was $12 \times 15.1 \mathrm{~cm}$ on the right and $15.4 \times 18.3 \mathrm{~cm}$ on the left (white arrow). CT, computed tomography.

history of coronary heart disease, diabetes mellitus and hypertension. No tumor history, no hereditary family history and no alcohol consumption were recorded.

On admission, the palpable mass around $10 \mathrm{~cm}$ was detected in the lower abdomen. It was a little hard and unmoved and there were no other positive signs. And the heart rate was 66 beats $/ \mathrm{min}$, the blood pressure was 120/70 mmHg. The level of the serum CA19-9 and CA125 were 489 and $179 \mathrm{U} / \mathrm{mL}$, respectively. The high CA19-9 level might indicate the primary pancreatic cancer, while the high CA125 level also implied the gynecological cancer. Therefore, the tumor antigen did not help to diagnose. No obvious abnormalities were detected during routine blood, biochemistry, and coagulation function tests. Abdominal ultrasound suggested solid-cystic lesions in the bilateral ovarian areas and a $3 \mathrm{~cm}$ solid lesion in pancreatic tail. Enhanced computed tomography (CT) revealed the hypoenhancing pancreatic mass with necrosis and vascular invasion, suggesting that the pelvic cavity was occupied by multilocular large cystic-solid lesions (Figure 1). The largest cross-sectional area was $12 \times 15.1 \mathrm{~cm}$ on the right side and $15.4 \times 18.3 \mathrm{~cm}$ on the left side, with a high possibility of malignancy. Considering the lesion on radiology and elevated tumor antigens, the pancreatic cancer or ovarian tumor was highly suspected. Then she undertook positron emission tomography CT (PET-CT) scan. The result revealed that there could be the cystadenocarcinoma in the pancreatic tail, invading the celiac trunk, with multiple metastatic lesions implanted in the pelvic.
Then final diagnosis was not clear with two possibilities, one was pancreatic cancer with ovarian metastases and the other one was two primary tumors. After multidisciplinary consultation, she underwent bilateral salpingooophorectomy and biopsy of the pancreatic tumor. The lesions showed a gray-pink multilocular cystic area with many cysts filled with clear yellow liquid (Figure $2 A$ ). The pathological examination suggested that the ovarian lesion was the metastatic cystadenocarcinoma, and the pancreatic tumor was diagnosed as the cystadenocarcinoma (T2N2M1) (Figure 2B,C). The immunohistochemical stain was as follows: CEA (+), CDX2 (+), CK20 (+), CK7 $(+)$, ER (-), P16 (-), p53 (+), WT-1 (-) and Vimentin (+). Gemcitabine $(1.4 \mathrm{~g} / \mathrm{dL}$ i.v.) and intraperitoneal perfusion chemotherapy with cisplatin $(50 \mathrm{mg})$ were given to the patient for nine cycles postoperatively. She developed mild myelosuppression during chemotherapy. The follow-up last for 26 months until she died.

All procedures performed in studies involving human participants were in accordance with the ethical standards of the institutional and/or national research committee(s) and with the Helsinki Declaration (as revised in 2013). Written informed consent was obtained from the patient.

\section{Discussion}

Pancreatic cystadenocarcinoma, a lethal malignant tumor with high mortality and extremely poor prognosis, has become one of the leading death causes among different 

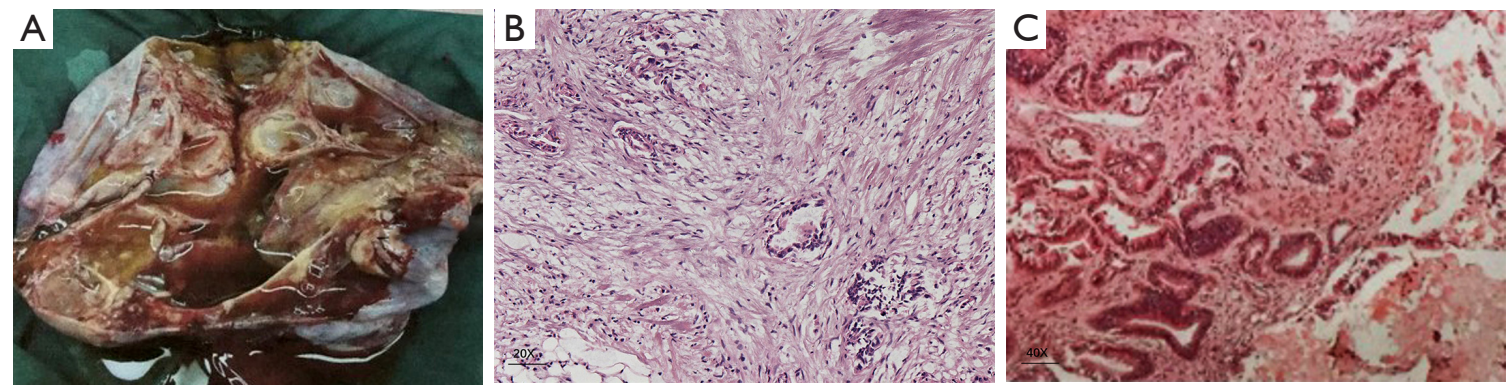

Figure 2 Gross specimen and immunohistochemistry. (A) The specimen of left ovary: the gray-pink multilocular cystic area with many cysts filled with clear yellow liquid. (B) Histopathological features pancreatic tumor. Hematoxylin and eosin staining. (C) Histopathological features ovarian tumor. Hematoxylin and eosin staining.

malignancies in the US (1). Besides, such cancer is difficult to be detected and diagnosed at the early stage. Additionally, pancreatic cancer combined with ovarian metastases is quite rare and even extremely difficult to diagnosis. Metastatic ovarian tumor secondary to gastrointestinal cancer is named as Krukenberg (4), but ovarian metastases from pancreas manifesting as Krukenberg has not been widely reported. In our case report, the patient manifested the lower abdominal mass and received tumor antigens and CT examination. But the limitation was that it failed to distinguish the original tumor. There was a dilemma whether a primary ovarian tumor or pancreatic cancer. Then she underwent PETCT which indicated the primary tumor might come from pancreas. Finally, the surgical operation was conducted after multidisciplinary consultation and revealed the truth.

Pancreatic primaries only take up approximately $7 \%$ of non-genital ovarian metastases (5). Furthermore, pancreatic metastatic cancer often invades bilateral ovaries, and the cancer emboli is usually found in lymphatic vessels during pathological test (6). Clinical presentation of these diseases usually lack the specificity. Most of the cases present as ovarian mass at the first time to hospital, which easy to disturb the accurate diagnosis of doctors. Some patients also show unresectable or local advanced pancreatic cancer with vascular invasion and distant metastases, sometimes losing the chance for pancreatectomy. Pre-operative serum level of CA125 can increase in ovarian cancers, but it is also elevated in a large portion of pancreatic cancer, which impair the specificity of ovarian cancer diagnosis $(7,8)$. Radiological method such CT and MRI could distinguish the pancreatic cancer and metastatic site efficiently $(9,10)$. PET/CT was also reported to diagnose ovarian metastasis as initial presentation of pancreatic carcinoma (11). Besides, endoscopic ultrasound-guided fine needle aspiration (EUS-
FNA) is safe in diagnosing solid pancreatic lesions $(12,13)$. It was reported that EUS-FNA with liquid-based cytology could diagnosis the metastatic small-cell carcinoma to the pancreas (13). In fact, surgeons and gynecologists tend to only focus on the pancreatic lesion or the ovarian neoplasm respectively, resulting in a missed diagnosis and delayed therapy. When pancreatic lesions and ovarian lesions were discovered synchronously, the suspicion of ovarian metastasis from pancreatic cancer should be considered in the differential diagnoses.

No optimal treatment strategy has been recommended for such kind of malignancies. Radiotherapy has no significant role in the prognosis of patients with Krukenberg tumor. And the effect of neoadjuvant or adjuvant chemotherapy for pancreatic cystadenocarcinoma is still unknown. Chemotherapy can prolong the survival of patients to some extent. Marco reported that gemcitabine plus oxaliplatin transform inoperable lesion to a resectable tumor in pancreatic mucinous cystadenocarcinoma with ovarian metastases (14). There is no doubt that surgical resection remains the curative method for cancer treatment. Even for metastasis cases, surgical operation can also prolong the survival time $(15,16)$. It is better to combine the pancreatectomy and the salpingo-oophorectomy, but palliative resection of ovarian masses can also benefit the patient, relieving the clinical symptoms (17). On the other hand, the differentiation of primary and metastasis ovarian cancer usually depends on the pathological results. Mucinous carcinoma of the ovary might suggest the presence of a non-ovarian primary tumor (18). Unfortunately, no immunohistochemical method can specifically distinguish the primary and metastatic ovarian tumors by itself nowadays. Adjuvant therapy should be given to patients according the 
pathological pattern with close follow-up. Generally, the prognosis of mucinous adenocarcinoma is better than that of the ductal adenocarcinoma in pancreas.

\section{Conclusions}

Considering that pancreatic cancer with ovarian metastases is relatively rare and easy to misdiagnosed, the doctors should pay more attention to the diagnosis and treatment of this disease. When the patient manifests tumors in pancreas and ovary concurrently, doctors cannot neglect the possibility of pancreatic cancer with ovarian metastases. Traditional preoperative imaging examination is beneficial for diagnosis. PET-CT and EUS-FNA contribute to differential diagnosis. Surgical operation is regarded as the effective treatment while aggressive surgery is not recommended as it does not extend life span. The pathological results finally make the correct diagnosis.

\section{Acknowledgments}

Funding: The study's design, collection, analysis, writing and interpretation were supported the project of application and promotion of capital special clinical research from Beijing Municipal Science \& Technology Commission (Z171100001017017018).

\section{Footnote}

Reporting Checklist: The authors have completed the CARE reporting checklist. Available at http://dx.doi.org/10.21037/ tcr-20-2361

Conflicts of Interest: All authors have completed the ICMJE uniform disclosure form (available at http://dx.doi. org/10.21037/tcr-20-2361). The authors have no conflicts of interest to declare.

Ethical Statement: The authors are accountable for all aspects of the work in ensuring that questions related to the accuracy or integrity of any part of the work are appropriately investigated and resolved. All procedures performed in studies involving human participants were in accordance with the ethical standards of the institutional and/or national research committee(s) and with the Helsinki Declaration (as revised in 2013). Written informed consent was obtained from the patient.
Open Access Statement: This is an Open Access article distributed in accordance with the Creative Commons Attribution-NonCommercial-NoDerivs 4.0 International License (CC BY-NC-ND 4.0), which permits the noncommercial replication and distribution of the article with the strict proviso that no changes or edits are made and the original work is properly cited (including links to both the formal publication through the relevant DOI and the license). See: https://creativecommons.org/licenses/by-nc-nd/4.0/.

\section{References}

1. Siegel RL, Miller KD, Jemal A. Cancer statistics, 2019. CA Cancer J Clin 2019;69:7-34.

2. Maitra A, Hruban RH. Pancreatic cancer. Annu Rev Pathol 2008;3:157-88.

3. Riley DS, Barber MS, Kienle GS, et al. CARE guidelines for case reports: explanation and elaboration document. J Clin Epidemiol 2017;89:218-35.

4. Kiyokawa T, Young RH, Scully RE. Krukenberg tumors of the ovary: a clinicopathologic analysis of 120 cases with emphasis on their variable pathologic manifestations. Am J Surg Pathol 2006;30:277-99.

5. Moore RG, Chung M, Granai CO, et al. Incidence of metastasis to the ovaries from nongenital tract primary tumors. Gynecol Oncol 2004;93:87-91.

6. Sakakura C, Hagiwara A, Yamazaki J, et al. Management of postoperative follow-up and surgical treatment for Krukenberg tumor from colorectal cancers. Hepatogastroenterology 2004;51:1350-3.

7. Bassi C, Salvia R, Gumbs AA, et al. The value of standard serum tumor markers in differentiating mucinous from serous cystic tumors of the pancreas: CEA, Ca 19-9, Ca 125, Ca 15-3. Langenbecks Arch Surg 2002;387:281-5.

8. Kim H, Kang KN, Shin YS, et al. Biomarker panel for the diagnosis of pancreatic ductal adenocarcinoma. Cancers (Basel) 2020;12:1443.

9. Hong SB, Choi SH, Kim KW, et al. Meta-analysis of MRI for the diagnosis of liver metastasis in patients with pancreatic adenocarcinoma. J Magn Reson Imaging 2020;51:1737-44.

10. Zhao R, Jia Z, Chen X, et al. CT and MR imaging features of pancreatic adenosquamous carcinoma and their correlation with prognosis. Abdom Radiol (NY) 2019;44:2822-34.

11. Hou G, Jiang Y, Li F, et al. Ovarian metastasis as initial presentation of pancreatic carcinoma on FDG PET/CT. Clin Nucl Med 2020;45:306-7. 
12. Hedenström P, Demir A, Khodakaram K, et al. EUSguided reverse bevel fine-needle biopsy sampling and open tip fine-needle aspiration in solid pancreatic lesions - a prospective, comparative study. Scand J Gastroenterol 2018;53:231-7.

13. Son JH, Park HK, Kim HS, et al. Endoscopic ultrasound-guided fine needle aspiration with liquidbased cytology preparation in the diagnosis of metastatic small-cell carcinoma in the pancreas. Diagn Cytopathol 2018;46:977-80.

14. Di Marco M, Vecchiarelli S, Macchini M, et al. Preoperative gemcitabine and oxaliplatin in a patient with ovarian metastasis from pancreatic cystadenocarcinoma. Case Rep Gastroenterol 2012;6:530-7.

15. Kubeček O, Laco J, Špaček J, et al. The pathogenesis,

Cite this article as: Wang SD, Dai MH, Zhao YP. Primary pancreatic cystadenocarcinoma with ovarian metastases in a 38-year-old female: case report. Transl Cancer Res 2020;9(12):76527656. doi: $10.21037 / \mathrm{tcr}-20-2361$ diagnosis, and management of metastatic tumors to the ovary: a comprehensive review. Clin Exp Metastasis 2017;34:295-307.

16. Al-Agha OM, Nicastri AD. An in-depth look at Krukenberg tumor: an overview. Arch Pathol Lab Med 2006;130:1725-30.

17. Falchook GS, Wolff RA, Varadhachary GR.

Clinicopathologic features and treatment strategies for patients with pancreatic adenocarcinoma and ovarian metastases. Gynecol Oncol 2008;108:515-9.

18. Lee KR, Young RH. The distinction between primary and metastatic mucinous carcinomas of the ovary: gross and histologic findings in 50 cases. Am J Surg Pathol 2003;27:281-92. 